\title{
Detection of BRAF V600E Mutation in Ganglioglioma and Pilocytic Astrocytoma by Immunohistochemistry and Real-Time PCR-Based Idylla Test
}

\author{
Justyna Durślewicz, ${ }^{1}$ Anna Klimaszewska-Wiśniewska ${ }^{1 D},{ }^{1}$ Paulina Antosik, ${ }^{1}$ \\ Anna Kasperska, ${ }^{1}$ Dariusz Grzanka, ${ }^{1}$ Tadeusz Szylberg, ${ }^{2}$ and Lukasz Szylberg ${ }^{1,2}$ \\ ${ }^{1}$ Department of Clinical Pathomorphology, Nicolaus Copernicus University in Toruń, Faculty of Medicine, Collegium Medicum \\ in Bydgoszcz, 85-092 Bydgoszcz, Poland \\ ${ }^{2} 10$ th Military Research Hospital and Polyclinic, 85-681 Bydgoszcz, Poland
}

Correspondence should be addressed to Anna Klimaszewska-Wiśniewska; ania.klimaszewska@op.pl

Received 3 April 2020; Revised 21 June 2020; Accepted 31 July 2020; Published 17 August 2020

Academic Editor: Hubertus Himmerich

Copyright (C) 2020 Justyna Durślewicz et al. This is an open access article distributed under the Creative Commons Attribution License, which permits unrestricted use, distribution, and reproduction in any medium, provided the original work is properly cited.

\begin{abstract}
The BRAF V600E mutation is an important oncological target in certain central nervous system (CNS) tumors, for which a possible application of BRAF-targeted therapy grows continuously. In the present study, we aim to determine the prevalence of BRAF V600E mutations in a series of ganglioglioma (GG) and pilocytic astrocytoma (PA) cases. Simultaneously, we decided to verify whether the combination of fully automated tests-BRAF-VE1 immunohistochemistry (IHC) and Idylla BRAF mutation assay-may be useful to accurately predict it in the case of specified CNS tumors. The study included 49 formalin-fixed, paraffin-embedded tissues, of which 15 were GG and 34 PA. Immunohistochemistry with anti-BRAF V600E (VE1) antibody was performed on tissue sections using the VentanaBenchMark ULTRA platform. All positive or equivocal cases on IHC and selected negative ones were further assessed using the Idylla BRAF mutation assay coupled with the Idylla platform. The BRAFVE1 IHC was positive in $6(6 / 49 ; 12.3 \%)$ and negative in 39 samples $(39 / 49 ; 79.6 \%)$. The interpretation of immunostaining results was complicated in 4 cases, of which 1 tested positive for the Idylla BRAF mutation assay. Therefore, the overall positivity rate was $14.3 \%$. This included 2 cases of GG and 5 cases of PA. Our study found that BRAF V600E mutations are moderately frequent in PA and GG and that for these tumor entities, IHC VE1 is suitable for screening purposes, but all negative, equivocal, and weak positive cases should be further tested with molecular biology techniques, of which the Idylla system seems to be a promising tool.
\end{abstract}

\section{Introduction}

According to the National Cancer Institute, tumors of the central nervous system (CNS) represent only a small fraction of annual tumor incidence (1.4\%) but are associated with a twice higher mortality rate $(2.8 \%)$. In children under 14 years old, CNS tumors are the most frequent solid tumors, and half of the cases occur in infants $0-4$ years old [1-3]. Brain tumors are a diverse group of neoplasms arising from different cells within the CNS or from primary tumors of other organs that spread to the CNS. Primary brain tumors include a number of histologic types with distinctly different tumor growth rates $[4,5]$.

Due to the uniqueness of the clinical material, we focused our attention on rare brain tumor types-ganglioglioma (GG) and pilocytic astrocytoma (PA). GGs are well-differentiated, rare CNS tumors, which are characterized by a slow, circumscribed growth and a relatively favorable prognosis. Gangliogliomas are generally benign WHO grade I tumors, most commonly located in the temporal lobes of children and young adults. However, they are both clinically and histologically heterogeneous, and tumor recurrence or anaplastic progression 
occurs in some cases [6]. PAs are a distinct histologic and biologic subset of gliomas that account for approximately $5.1 \%$ of all these tumors. It is the most common pediatric brain tumor in children [7]. Although preferentially located in the cerebellum, PA can arise anywhere in the CNS. Almost all are generally considered WHO grade I tumors [8].

One of the mutations that has aroused considerable interest in recent years concerns the $B R A F$ (v-raf murine sarcoma viral oncogene homolog $B$ ) gene that encodes the protein belonging to a highly oncogenic RAS/RAF/MEK/ERK signaling pathway [9]. The serine/threonine protein kinase BRAF is an important player in the mitogen-activated protein kinase (MAPK) signaling pathway that transduces mitogenic signals from activated cell-surface growth factor receptors to the cell nucleus and as a result modulates many important cellular processes, such as tumor growth, differentiation, proliferation, and angiogenesis. Deregulation of these processes by oncogenic BRAF has been implicated in different mechanisms underlying cancer development and progression. Therefore, the BRAF pathway has become a molecular target for individualized cancer therapy, with promising results deriving from clinical trials [10].

The most frequent mutation is a single nucleotide substitution of thymine to adenine at nucleotide 1799 that converts valine (V) to glutamic acid (E) at amino acid 600 (V600E mutation) [11]. Other common types of BRAF mutations in codon 600 are BRAF V600E2, V600K, V600D, V600R, and V600M4 [12]. An alternative mechanism of MAPK activation is the formation of BRAF fusion genes. The most common fusion is between exon 16 of KIAA1549 and exon 9 of $B R A F$, with less frequent fusion variants, including exon 16-exon 11 and exon 15-exon 9 [10, 13]. Currently, the KIAA1549-BRAF fusion accounts for around $58-75 \%$ of PA cases, so it is the most prevalent genetic alteration in this tumor entity. BRAF fusion results from tandem duplications or deletions on chromosome arms 7q.34 [3, 14-17].

The studies revealed that BRAF V600E mutation occurs in $100 \%$ of hairy cell leukemia [18], $50-60 \%$ of unresectable and metastatic malignant melanomas [19], approximately $30-50 \%$ of papillary thyroid carcinomas [20], 38\% of Langerhans cell histiocytosis [21], 15-35\% of serous low-grade and borderline ovarian carcinomas [22], 5-15\% of colorectal adenocarcinomas [22], and 3-5\% of non-small-cell lung carcinoma [9, 17-24]. Furthermore, BRAF mutations play an important role also in neurooncology [25]. The BRAF V600E mutation was observed in CNS tumors, e.g., in 66\% of pleomorphic xanthoastrocytoma [26], 51\% of dysembryoplastic neuroepithelial tumor [27], 18-57\% of ganglioglioma [28], $5-15 \%$ of pilocytic astrocytoma $[16,17]$, and $1 \%$ of glioblastoma [26].

A crucial part of therapeutic strategies is rapid detection of a mutant protein. The immunohistochemical detection of the BRAF V600E mutation is possible using an antibody of choice. An alternative route is the use of existing molecular biology techniques to analyze point and other mutations in the $B R A F$ gene. Among these techniques, the Idylla mutation tests coupled with the Idylla platform (Biocartis) have recently been proposed to serve as an attractive tool for the fast and easy detection of therapeutic markers, including
$B R A F$. The Idylla BRAF mutation test is a fully automated, real-time PCR-based molecular diagnostics system, able to identify the presence of $\geq 1 \% B R A F$ V600E/E2/D/K/R/Mmutated cells in formalin-fixed, paraffin-embedded (FFPE) tumor tissue samples. Access to the sensitive and specific diagnostic tests and reliable tools to detect mutant proteins and genes is helpful in the implementation of appropriate therapeutic strategies.

Although in the case of certain CNS tumors the relevance of BRAF V600E mutation in the clinical setting has been increasingly acknowledged, a relatively low frequency of its occurrence requires further investigations and multiple experimental cohorts to establish its mutational status as a definitive biomarker for these tumors. Therefore, the primary aim of this study was to determine BRAF V600E mutation status in patients with rare CNS tumors-ganglioglioma and pilocytic astrocytoma. In addition, given that there is no standard method for testing $B R A F$ status in diagnostic neurooncology [17], we aim to verify whether the combination of fully automated tests that are highly accessible for pathology laboratories-BRAF-VE1 IHC on VentanaBenchMark ULTRA platform and real-time PCR-based Idylla BRAF mutation assay-may be useful to accurately predict it in the FFPE tissues of GG and PA.

\section{Material and Methods}

2.1. Material. Archived FFPE tissues derived from 49 patients with tumors of the central nervous system, who were operated on in the 10th Military Research Hospital and Polyclinic, Bydgoszcz, Poland, between 2013 and 2018, were included in the present study. Histopathological diagnosis for each tumor sample was performed by two independent pathologists in the Department of Clinical Pathology, Collegium Medicum in Bydgoszcz, and in the Department of Pathology, 10th Military Research Hospital and Polyclinic. The study includes in total 15 gangliogliomas and 34 pilocytic astrocytomas. The median age at diagnosis was 28 (range: 9 -76), and the overall male:female ratio was $25: 24$. There were 4 pediatric patients ( $<18$ years; median age 15 , range: 9-17), and others were adults ( $\geq 18$ years, median age 28 , range: 18-76). Representative tumor areas were selected in order to perform immunohistochemical and molecular tests on FFPE specimens.

2.2. Ethics Statement. The study protocol has been approved by The Ethics Committee of Nicolaus Copernicus University in Toruń, Ludwik Rydygier Collegium Medicum in Bydgoszcz (approval number KB 737/2019).

\subsection{Methods}

2.3.1. Immunohistochemistry. Selected paraffin blocks were cut using a manual rotary microtome (Accu-Cut, Sakura, Torrance, CA, USA) to $3.0 \mu \mathrm{m}$ thick paraffin sections, placed on extra adhesive slides (SuperFrost Plus; Menzel-Glaser, Braunschweig, Germany), and dried at $60^{\circ} \mathrm{C}$ for $1 \mathrm{~h}$. Subsequently, deparaffinization and rehydration were performed in EZ Prep solution (Ventana Medical Systems, Tucson, AZ, USA). Next, antigen retrieval of tissue sections was 
TABLE 1: BRAF mutations detected by the Idylla BRAF mutation test.

\begin{tabular}{|c|c|c|c|c|c|}
\hline Exon & Codon & Mutation & Nucleotide change & Protein & Genetic call \\
\hline \multirow{6}{*}{15} & \multirow{3}{*}{600} & V600E & p.(Val600Glu) & $(\mathrm{c} .1799 \mathrm{~T}>\mathrm{A})$ & \multirow{3}{*}{$\mathrm{V} 600 \mathrm{E} / \mathrm{E} 2 / \mathrm{D}$} \\
\hline & & V600E2 & p.(Val600Glu) & (c.1799_1800delinsAA) & \\
\hline & & V600D & p.(Val600Asp) & (c.1799_1800delinsAT; c.1799_1800delinsAC) & \\
\hline & \multirow{3}{*}{600} & V600K & p.(Val600Lys) & $($ c.1798_ 1799delinsAA $)$ & \multirow{3}{*}{$\mathrm{V} 600 \mathrm{~K} / \mathrm{R} / \mathrm{M}$} \\
\hline & & V600R & p.(Val600Arg) & (c.1798_ 1799delinsAG) & \\
\hline & & V600M & p.(Val600Met) & $(\mathrm{c} .1798 \mathrm{G}>\mathrm{A})$ & \\
\hline
\end{tabular}

performed in a high $\mathrm{pH}$ Cell Conditioning (CC1) solution for $64 \mathrm{~min}$. Immunohistochemical staining was done using the BenchMark ${ }^{\circledR}$ ULTRA automated slide processing system (Ventana Medical Systems, Tucson, AZ, USA) and visualized using the OptiView DAB IHC Detection Kit (Ventana Medical Systems, Tucson, AZ, USA), as recommended by the manufacturer. Incubation with the primary antiBRAFV600E (VE1) antibody (Ventana Medical Systems, Tucson, AZ, USA) was performed for $40 \mathrm{~min}$ at $36^{\circ} \mathrm{C}$. The slides were incubated with OptiView HQ Universal Linker and OptiView HRP Multimer and counterstained in hematoxylin and bluing reagent. Finally, the sections were dehydrated in increasing ethanol concentrations $(80,90,96$, and $99.8 \%$ ), cleared in xylenes (I-IV), and sealed using a Dako mounting medium (Perlan, Inc., Santa Clara, CA, USA). Immunoreaction was labeled positive when unambiguous cytoplasmic staining of uniform or near-uniform intensity was seen and negative in the absence of staining or in the case of nuclear staining or when a staining of nontumor cells was observed. Of positive cases, the intensity of staining was scored as weak $(1+)$, moderate $(2+)$, or strong $(3+)$. The criteria for equivocal immunoreaction included a faint staining indistinguishable from a nonspecific background staining, as well as highly heterogeneous cytoplasmic staining. The latter was defined as the presence of distinct subpopulations of tumor cells having a staining intensity that differed up to two scoring levels, e.g., one population of tumor cells with 3 + and another with 0 (negative). The scores for positive immunoreactivity were categorized as follows: $0: 0 \%$ of stained cell/area; $11-25 \%$ of stained cells/area; $226-50 \%$ of stained cells/area; $351-75 \%$ of stained cell/area; and 4 equal or more than $76 \%$ of stained cells/area.

2.3.2. Idylla BRAF Mutation Test. Using the automated Idylla $^{\mathrm{TM}}$ molecular diagnostics platform (Biocartis, Mechelen, Belgium), the selected specimens were tested for mutations in codon 600 of the BRAF gene. The Idylla ${ }^{\mathrm{TM}}$ platform is a realtime PCR and fluorophore-based detection system. Prior to the analysis, each sample was verified by independent pathologists, to confirm that at least $30 \%$ of tumor cells were present in every section. Selected paraffin blocks were cut using the manual rotary microtome (Accu-Cut; Sakura) to $10 \mu \mathrm{m}$ thick paraffin sections, which were placed between qualitative filter papers ( $10 \mathrm{~mm}$ in diameter), and then inserted to the Idylla ${ }^{\mathrm{TM}}$ BRAF mutation test cartridge (CE-IVD approved). For FFPE specimens, the sample preparation module uses high-intensity focused ultrasound technology to emulsify the paraffin and simultaneously rehydrate the tissue sample in an aqueous solu- tion. Subsequently, isolated nucleic acids are transferred via microfluidic channels of the cartridge to appropriate separate PCR chambers with predeposited PCR reagents (i.e., primers, probes, and enzymes). To provide appropriate real-time PCR amplification and detection, all reagents are used in a dry form. Each PCR chamber allows for the identification of up to 6 different biomarker groups, each of which can be composed of multiple individual biomarkers. Once the sample is inserted into the cartridge and the lid is closed, the cartridge is hermetically sealed to eliminate risk of PCR contamination. The results, calculated by the dedicated Idylla ${ }^{\mathrm{TM}}$ software, were available after a $90 \mathrm{~min}$ run time. The test consists of three allele-specific PCR reactions that enable identification of $B R A F$ wild-type, $B R A F$ $\mathrm{V} 600 \mathrm{~K} / \mathrm{R} / \mathrm{M}$ (all c.1798G $>\mathrm{A}$ ), or BRAF V600E/E2/D (all c.1799T $>$ A) sequences (Table 1). The test enables identifying the presence of $\geq 1 \%$ BRAF V600 mutation in a background of wild-type allele. A quantification cycle $(\mathrm{Cq})$ value is calculated by Idylla ${ }^{\mathrm{TM}}$ software for every valid PCR curve. The presence of a mutant genotype was determined based on the difference between $\mathrm{Cq}$ for wild-type $B R A F$ and the $\mathrm{V} 600 \mathrm{E} / \mathrm{E} 2 / \mathrm{D}$ or V600K/R/M Cq values. BRAF total, i.e., wild-type gene, was used as a sample processing control, and the melanoma specimen with established BRAF mutation served as a positive control. The mutant signal is considered valid if the $\triangle \mathrm{Cq}$ is within a validated range. BRAF V600 mutation-negative samples were those for which a valid wild-type signal was observed but a $\Delta \mathrm{Cq}$ value was outside the validated range $[29,30]$. The interpretation of results is fully automatic with 4 possible results on the screen of the Idylla console: (i) no mutation detected in $B R A F$ codon 600 , (ii) mutation detected in $B R A F$ codon 600 , (iii) insufficient DNA input, and (iv) invalid.

\section{Results}

All 49 FFPE samples were examined for the presence of BRAF V600E mutation by IHC using the anti-BRAF V600E (VE1) antibody with the OptiView DAB IHC detection kit and the automated VentanaBenchMark ${ }^{\circledR}$ ULTRA platform. Of these samples, $6(6 / 49 ; 12.3 \%)$ were scored as BRAF-VE1-positive, 4 $(4 / 49 ; 8.2 \%)$ as equivocal, and $39(39 / 49 ; 79.6 \%)$ as negative. Staining intensity was strong in $2(2 / 6 ; 33,3 \%)$ cases, moderate in $3(3 / 6 ; 50 \%)$ cases, and weak in $1(1 / 6 ; 16.7 \%)$ sample. Half of the equivocal cases were GGs, and the other half were PAs. Both ambiguous cases of GG (nos. 2 and 3) were characterized by a small tumor cell content (30 and 10\%) and cytoplasmic staining that was difficult to distinguish from nonspecific background staining. A similar situation, with respect to staining intensity, but not tumor cellularity (95\%) was observed in one 


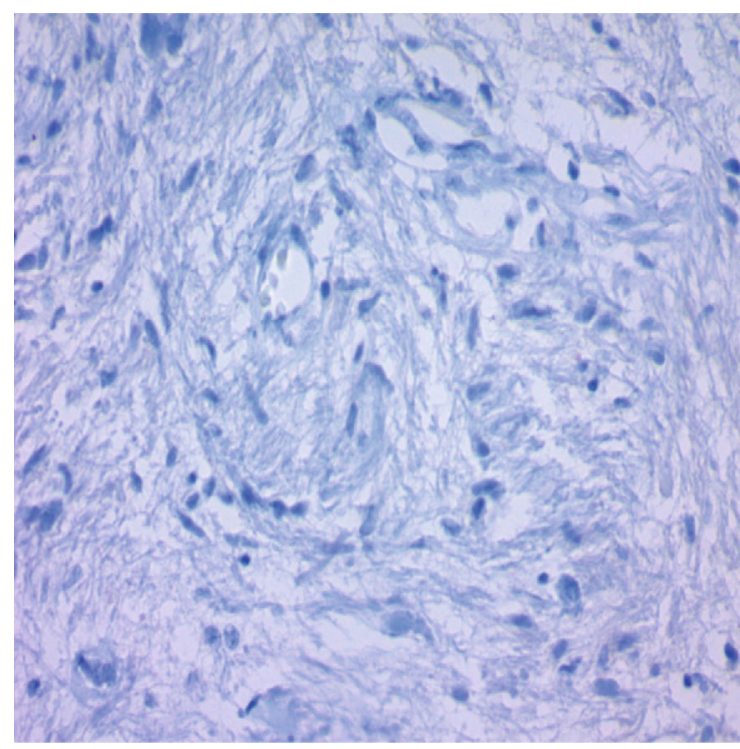

(a)

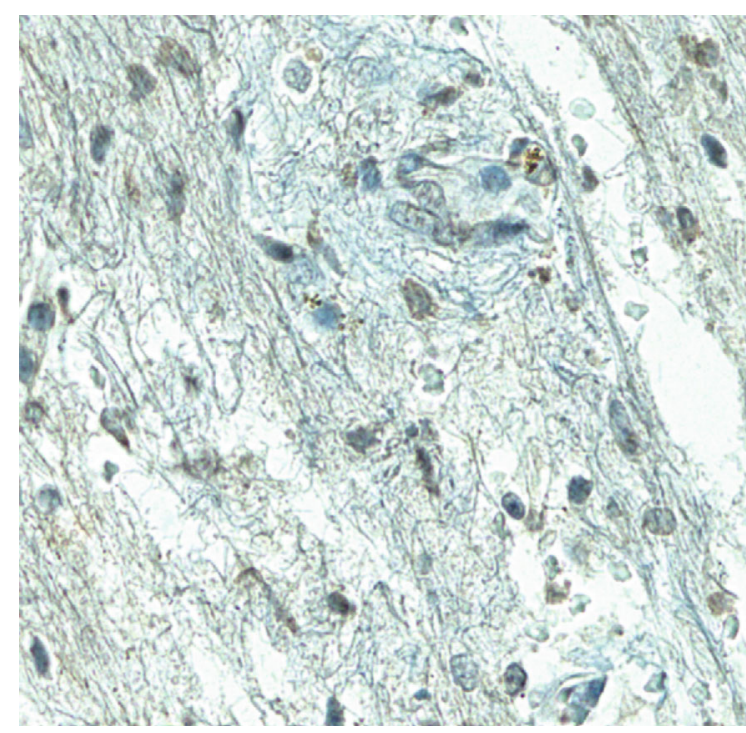

(c)

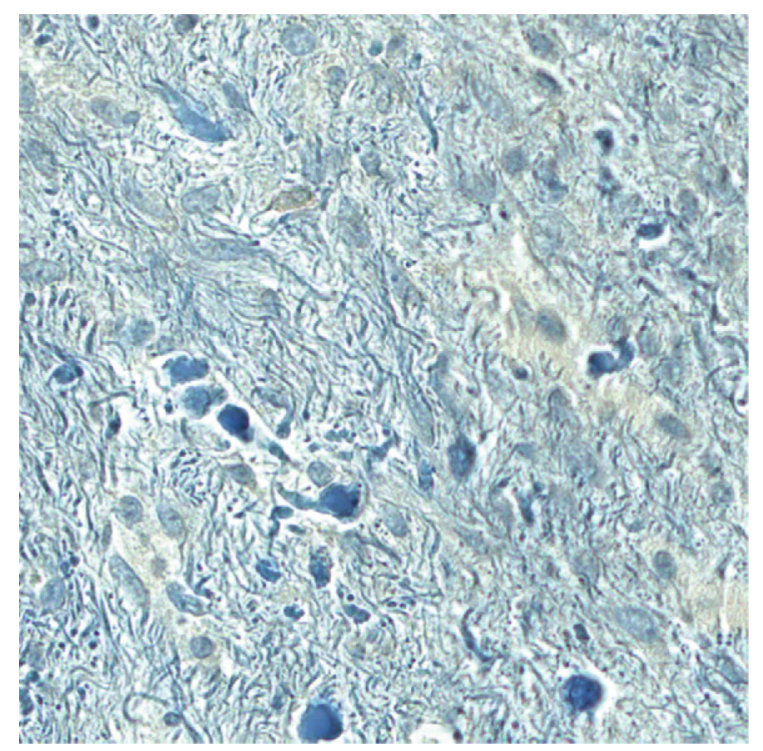

(b)

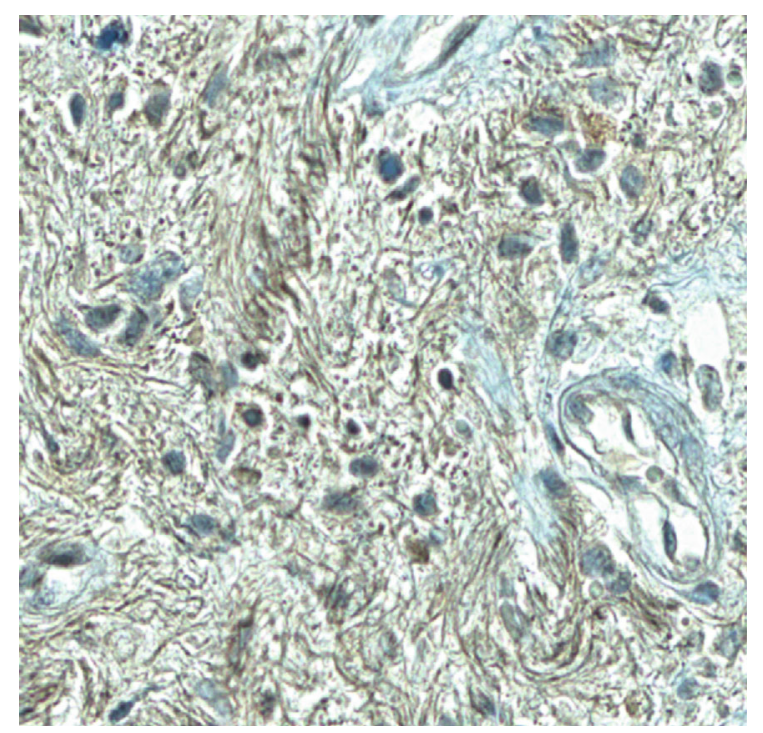

(d)

FIGURE 1: Immunohistochemical staining pattern of BRAF V600E (VE1) protein and negative control of the same case. Highly heterogeneous staining pattern is seen for PA case (no. 10), with different binding intensity-from clearly VE1-negative (b) tumor cells, through weak (c), to even strong (d). The image (a) of the same case stained with negative control is also seen. Magnification: 400x.

PA sample (no. 9). The other PA case (no. 10) was particularly complicated to interpret, due to a highly heterogeneous staining pattern. In these sections, there were the nests of VE1-positive tumor cells, with almost every subpopulation showing a different binding intensity-from weak to even strong, in addition to clearly VE1-negative (staining intensity 0 ) tumor cells (Figure 1). In addition, nonspecific nuclear staining along with cytoplasmic staining could be occasionally seen in tumor cells (Figure 2).

All cases positive or equivocal for BRAF V600E testing on immunohistochemistry were further assessed on the Idylla ${ }^{\mathrm{TM}}$ platform using the Idylla ${ }^{\mathrm{TM}}$ BRAF mutation assay. Of 6 positive BRAF-VE1 cases, all tested positive for $B R A F$ $\mathrm{V} 600 \mathrm{E} / \mathrm{E} 2 / \mathrm{D}$ mutation and none were positive for $B R A F$ $\mathrm{V} 600 \mathrm{~K} / \mathrm{R} / \mathrm{M}$ mutation. Among these positive specimens, there was $1(1 / 15 ; 6.7 \%)$ case of ganglioglioma and $5(5 / 34$; $14.7 \%)$ cases of pilocytic astrocytoma. Of 4 equivocal BRAF-VE1 samples, only 1 (no. 2) tested positive for $B R A F$ V600E/E2/D mutation on the Idylla real-time PCR BRAF mutation test, whereas others were negative (each sample repeated twice). In addition, $12(12 / 39 ; 30.8 \%)$ randomly selected cases from VE1-negative tumor samples, were further subjected to the Idylla ${ }^{\mathrm{TM}} B R A F$ mutation test. The molecular analysis confirmed their negative mutation status, with respect to not only BRAF V600E/E2/D mutations but also $B R A F \mathrm{~V} 600 \mathrm{~K} / \mathrm{R} / \mathrm{M}$ mutations. Therefore, the overall positivity rate found in our study was $14.3 \%(7 / 49)$. This included 2 $(2 / 15 ; 13.3 \%)$ cases of ganglioglioma and $5(5 / 34 ; 14.7 \%)$ cases of pilocytic astrocytoma. The IHC-positive or equivocal cases ran on the Idylla real-time PCR instrument for the 


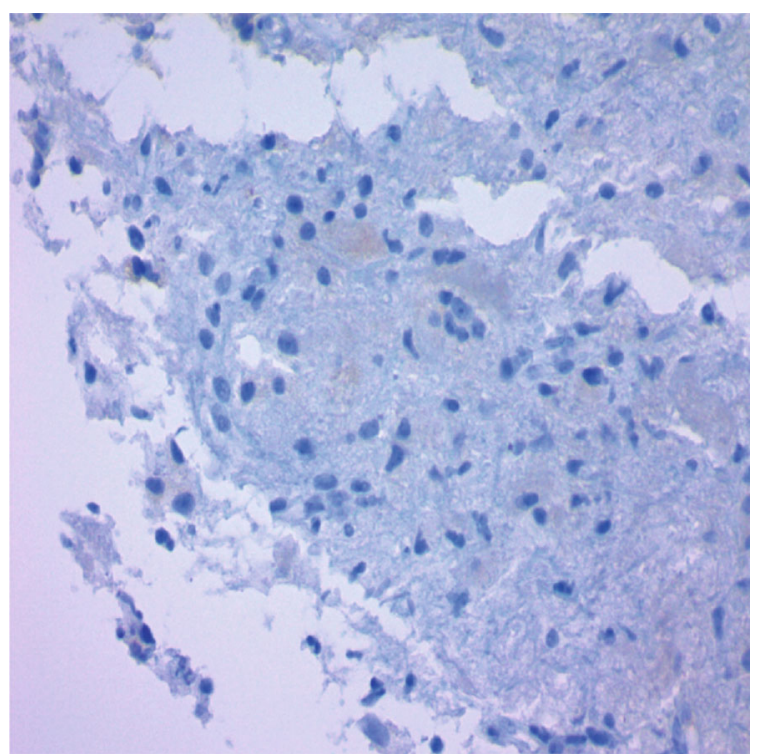

(a)

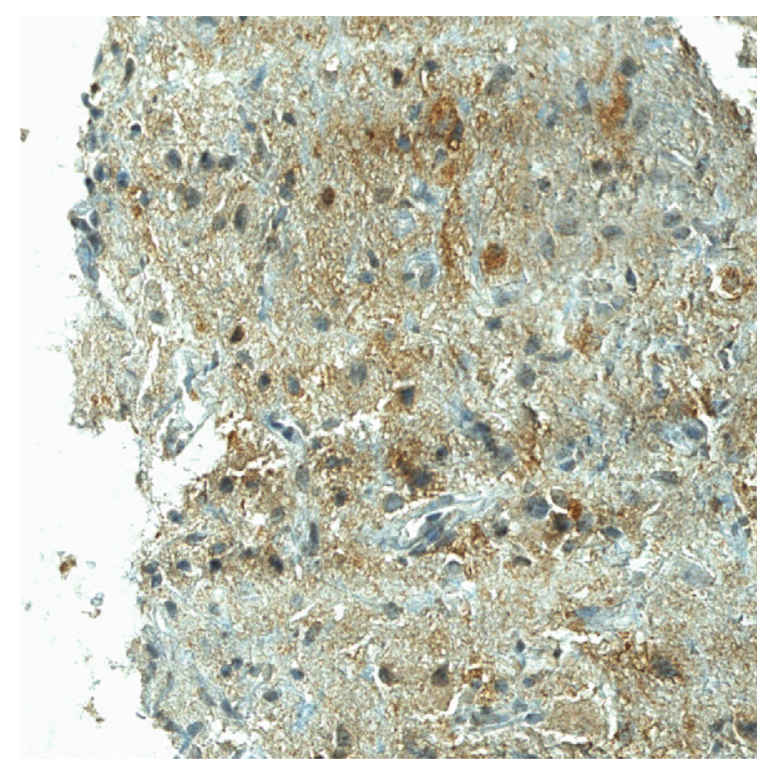

(b)

FIGURE 2: Negative control and immunohistochemical staining pattern of BRAF V600E (VE1) protein of the same case. Negative control (a) and nonspecific nuclear staining along with cytoplasmic staining in tumor cells (b) are seen. Magnification: 400x.

TABLE 2: BRAF V600 mutations detected or not by Idylla ${ }^{\mathrm{TM}}$ in BRAF-VE1-positive or equivocal cases.

\begin{tabular}{|c|c|c|c|c|c|c|c|c|}
\hline $\begin{array}{l}\text { Sample } \\
\text { no. }\end{array}$ & $\begin{array}{l}\text { Histopathological } \\
\text { diagnosis }\end{array}$ & Sex & Age & Tumor location & $\begin{array}{l}\text { IHC staining } \\
\text { intensity }\end{array}$ & $\begin{array}{c}\text { Category of } \\
\% \\
\text { stained cells }\end{array}$ & IRS & BRAF V600E Idylla \\
\hline 1 & $\begin{array}{l}\text { Ganglioglioma } \\
\text { (WHO grade I) }\end{array}$ & M & 23 & Hippocampus & $2+$ & 4 & 8 & V600E/V600E2/V600D \\
\hline 2 & $\begin{array}{l}\text { Ganglioglioma } \\
\text { (WHO grade I) }\end{array}$ & M & 51 & Left temporal lobe & \multicolumn{2}{|c|}{ Equivocal } & & V600E/V600E2/V600D \\
\hline 3 & $\begin{array}{l}\text { Ganglioglioma } \\
\text { (WHO grade I) }\end{array}$ & M & 47 & Left frontal lobe & \multicolumn{2}{|c|}{ Equivocal } & & WT \\
\hline 4 & $\begin{array}{l}\text { Pilocytic astrocytoma } \\
\text { (WHO grade I) }\end{array}$ & $\mathrm{F}$ & 31 & Left hemisphere & $1+$ & 4 & 4 & V600E/V600E2/V600D \\
\hline 5 & $\begin{array}{l}\text { Pilocytic astrocytoma } \\
\text { (WHO grade I) }\end{array}$ & M & 18 & Left temporal lobe & $2+$ & 4 & 8 & V600E/V600E2/V600D \\
\hline 6 & $\begin{array}{l}\text { Pilocytic astrocytoma } \\
\text { (WHO grade I) }\end{array}$ & M & 18 & Left temporal lobe & $2+$ & 3 & 6 & V600E/V600E2/V600D \\
\hline 7 & $\begin{array}{l}\text { Pilocytic astrocytoma } \\
\text { (WHO grade I) }\end{array}$ & $\mathrm{F}$ & 21 & Cerebellar vermis & $3+$ & 3 & 9 & V600E/V600E2/V600D \\
\hline 8 & $\begin{array}{l}\text { Pilocytic astrocytoma } \\
\text { (WHO grade I) }\end{array}$ & M & 22 & Left temporal lobe & $2+$ & 4 & 8 & V600E/V600E2/V600D \\
\hline 9 & $\begin{array}{l}\text { Pilocytic astrocytoma } \\
\text { (WHO grade I) }\end{array}$ & M & 31 & Right temporal lobe & \multicolumn{2}{|c|}{ Equivocal } & & WT \\
\hline 10 & $\begin{array}{l}\text { Pilocytic astrocytoma } \\
\text { (WHO grade I) }\end{array}$ & M & 31 & Lateral ventricle & \multicolumn{2}{|c|}{ Equivocal } & & WT \\
\hline
\end{tabular}

confirmation, and further assessment of BRAF V600 status is presented in Table 2.

F: female; M: male; IHC: immunohistochemistry; V600E/V600E2/V600D: presence of BRAF-V600E mutation detected by Idylla ${ }^{\mathrm{TM}}$; WT: wild type; IRS: the immunoreactive score of Remmele and Stegner, category of stained cells: 0: 0\% of stained cell/areas; 1 : 1-25\% of stained cells/area; $2: 26-50 \%$ of stained cells/area; $3: 51-75 \%$ of stained cell/area; 4 : equal or more than $76 \%$ of stained cells/area.

\section{Discussion}

The BRAF V600E mutation, among other molecular aberrations of this gene (in particular the presence of KIAA1549- 
$B R A F$ fusions), is found in several CNS tumors, including gliomas and glioneuronal tumors. Although prognostic significance of BRAF alterations for these tumor entities is still inconclusive, a diagnostic utility for the differential diagnosis of pediatric gliomas, as well as a possible therapeutic utility, makes the assessment of their occurrence of growing clinical relevance [16]. Indeed, more and more case reports and ongoing clinical trials have been showing that CNS cancer patients harboring BRAF V600 mutations are responsive to BRAF (and MEK) inhibitors [31-33]. Therefore, it seems now clear that the diagnosis of CNS tumors should include molecular testing for BRAF mutations or fusions, and, if present, patients ought to be considered for targeted treatment [34].

The frequency of BRAF V600E mutations in GG and PA varies across scientific reports from 18 to $57 \%$ and from 5 to $15 \%$, respectively $[16,17,28]$. However, in this topic, there is a relatively limited number of studies dedicated to these rare tumor types, compared with the multitude of research on, e.g., melanoma or papillary thyroid carcinoma. Hence, it was reasonable to assess BRAF V600E prevalence in an additional cohort. In relation to the largest study by Schindler et al. [35], who analyzed 1320 CNS tumor cases of pediatric and adult patients, including 77 GGs and 97 PAs of which $18 \%$ and $9 \%$ carried BRAF V600E mutation, we demonstrated a quite similar incidence rate, namely, $13.3 \%$ and $14.7 \%$, respectively. This small but noticeable discrepancy may be due to the size of the research group, given that the small number of patients is undoubtedly a limitation of our study. However, a marked discordance exists between our results and those reported by Chappé et al. [36], Dougherty et al. [37], and Dahiya et al. [32], who observed the presence of BRAF V600E mutation in 45.2\% (14/31), 50\% (9/18), and $38.3 \%(18 / 47)$ of pediatric gangliogliomas, respectively. A high frequency of BRAF V600E mutations (58\%) has been also revealed by Koelsche et al. [38] in a series of 71 pediatric and adult cases of GG (median age 22 years, range: 5-69). Importantly, they have demonstrated that BRAF V600E mutation is strongly associated with younger patient age and shows the highest frequency in the first decade of life, gradually decreasing thereafter. More specifically, GG patients with BRAF V600E mutations had a median age of 19 (range: 5-52) years at surgery, whereas patients with $B R A F$ wild-type status were significantly older with a median of 31 years (range: 12-69). A similar age-dependent incidence of BRAF V600E mutations in GGs has been observed by Gierke et al. [16]. Furthermore, Myung et al. [39] have also demonstrated that the mutation was more common in pediatric GGs (34.7\%) than adult counterparts (14.3\%), but this relationship did not reach a statistical significance [39]. Our cohort included mostly adult patients $(91.8 \%$ of cases, median age 28 years, range: 18-76); hence, discrepancies as to the frequency of BRAF mutations between the cited findings $[17,32,36]$ and these presented here may be also related to this fact, apart from an obvious reason related to a low number $(n=15)$ of GG cases. In turn, a different pattern [16] or no association between patient age and BRAF V600E mutation $[35,40]$ has been found for pilocytic astrocytoma. In this context, Gierke et al. have shown that
V600E mutations in PA were very scarce in the pediatric age group $(2 \% ; 1 / 45)$ and were limited to the middle age group $(13 \% ; 3 / 23)$ [16]. This could at least partially explain a higher rate of positive PA cases in our cohort as compared to the positivity rates previously reported in most of other studies (usually below $10 \%)[35,40]$. Our result $(14.7 \%$; $5 / 34)$ is close to the mutation rate $(15.6 \% ; 7 / 45)$ reported by Myung et al. [39] who, in addition, have shown that the presence of this mutation was not significantly related to patient age but was slightly more frequent in adult patients (17.6\%) than pediatric ones (14.3\%). Apart from different sample sets (varied in terms of, e.g., group size and age of patients), it should also be noted that the variations in the frequency of BRAF mutations between different studies, to the same extent, may also be attributed to methodology and equipment used.

The gold standard for BRAF mutation analysis is molecular biology (DNA-based) techniques, with classical Sanger sequencing being the most commonly used [41, 42]. However, in the case of CNS tumors, in particular ganglioglioma, the relevance of the latter method has recently been questioned [40,43], due to its high detection threshold of $20 \%$ allele frequency, which does not work well with a low number and/or scattered distribution of neoplastic cells [44]. Although other molecular biology techniques, such as pyrosequencing, next-generation sequencing, PNA-clamping PCR, ddPCR, and ASqPCR, have higher sensitivity (detection of $0.02-10 \%$ mutant in a background of wild type), all DNA-based methods are often expensive, labor-intensive, time-consuming, and not widely available in pathology laboratories, also because they require a highly skilled operator and complex infrastructure $[45,46]$. Immunohistochemistry with a recently developed mouse monoclonal mutationspecific antibody (VE1 cone) has shown promise as a more widely available, relatively inexpensive, and fast method, which allows a direct visualization of BRAF V600E protein at the single-cell level $[47,48]$. Numerous studies on melanoma and papillary thyroid carcinoma have demonstrated an excellent concordance between VE1 IHC and molecular analysis (a sensitivity and specificity over 95\%) [49-52]. However, the sensitivity and specificity of VE1 IHC in colorectal cancer range from 59 to $100 \%$ and 51 to $100 \%$, respectively, and therefore, some of the studies concluded that this method should not be used to guide patient management in this disease entity [53]. In the case of CNS tumors, VE1 IHC was evaluated in several studies, in which it has been presented as an either excellent or suboptimal [54] technique in characterizing brain tumor tissue $[25,26,40,55]$.

Although sometimes burdened with some technical difficulties, the VE1 antibody has been suggested to be even superior to the sole use of sequencing in these tumor entities (especially GG). The reason is that VE1 immunostaining can be performed as part of a clinical routine, and it has been shown to detect BRAF V600E mutant GGs in sequencingnegative cases $[40,43]$. Based on our results and those reported by other groups $[54,56]$, it seems that strong-tomoderate staining for the VE1 antibody in CNS tumors equates with the positive results of BRAF V600E molecular testing. Although in our study, this was also true for weak 
(1+) positive VE1 case, we feel that in daily practice such cases should be also labeled as those that require confirmation with molecular methods. It is mostly due to a nonspecific background staining that could be seen by us and others [54] with VE1 antibody in brain tumor tissues, making the interpretation of staining acquired on the sample ambiguous. In turn, no additional confirmation for BRAF V600E mutation appears necessary with completely negative VE1 results, as our randomly selected IHC-negative cases were also negative by molecular analysis. However, a lack of immunostaining does not rule out other mutation types at hotspot codon 600 , which yet have a potential clinical implications, as patients with mutations other than V600E variant are also eligible to BRAF-targeted therapies [57]. In our study, we found 3 equivocal samples due to low staining intensity. Another sample was labeled "equivocal" based on strongly heterogeneous staining, which has also been previously described in the case of VE1 antibody in colon tumors, as the staining pattern recommended for proceeding to mutational assessment by molecular methods [48]. Only 1 of these IHC-equivocal cases tested positive for BRAF V600E mutation (no. 2). This was ganglioglioma, in which it was not possible to distinguish a weak immunostaining from a background staining. Thus, our study clearly shows that there is sometimes a thin line between a weak genuine VE1 immunostaining and nonspecific background staining, and taking into account the subjectivity of the examiners and their varied experience level, as well as the fact that this antibody recognizes only $\mathrm{V} 600 \mathrm{E}$ variant, we suggest that the weak positive, equivocal, and negative cases on IHC should be further tested with molecular biology techniques. Simultaneously, this seems to be a good approach in scientific research, given that there are studies assuming a weak positive VE1 staining $(1+)$ as negative $[41,58]$. Collectively, our results highlight the importance of pursuing the occasional weak and equivocal results to further classification of mutational status. Therefore, we join the recent opinion of some authors that the utility of VE1 IHC in routine neuropathology should be limited to screening purposes [26]. This is also concordant with the recent recommendations for colorectal cancer and melanoma [41].

Given that BRAF-VE1 IHC in CNS tumor samples should be used in conjunction with molecular analysis, and that, as mentioned above, a conventional direct sequencing has a limited power in some of these samples, we decided to verify whether the Idylla $B R A F$ mutation assay performed on the fully automated Idylla platform may be a useful tool to accurately predict $B R A F$ mutation status in GG and PA. It seemed particularly justifiable in the case of these peculiar tumor tissues since the Idylla test has a benefit of high sensitivity with $1 \%$ detection limit, and simultaneously, it can be easily performed within approximately $90 \mathrm{~min}$ (with a hands-on time of less than $2 \mathrm{~min}$ ), as part of a clinical routine in all pathology units without the necessity of additional staff with molecular biology expertise or extramolecular infrastructure. This is due to the fact that the Idylla technology is based on disposable cartridges that integrate all preprocessing steps (deparaffinization, tissue digestion, and DNA extraction), PCR thermocycling, and fluorescence detection in a single diagnostic test with all reagents on-board and an automated setup workflow. In addition, no posttest analysis is required, since the Idylla console autoanalyzed the PCR curves to qualitatively determine the $B R A F$ mutation status $[59,60]$.

The Idylla BRAF mutation assay performed on the Idylla molecular diagnostics platform is an allele-specific real-time PCR-based technique, which has got CE-IVD certification for the qualitative detection of the V600E/E2/D and V600K/R/M mutations in FFPE samples of malignant melanoma [61-64]. It was also validated in thyroid tumor tissues [65] and colorectal cancer tissues [66]; however, to our knowledge, it has previously been tested only in few samples of CNS tissue [61, 67] but not in the case of GG tissues. In our study, all cases with positive results for BRAF V600E protein expression on IHC were tested positively using the Idylla ${ }^{\mathrm{TM}}$, which confirms the effectiveness of this system. Importantly (as mentioned above), we could also detect a BRAF V600E mutation in our IHC-equivocal FFPE sample (no. 2) that contained $30 \%$ tumor cells and, as such, did not meet the minimum tissue requirement for the Idylla $B R A F$ mutation testing [60]. In the other 3 IHC-equivocal cases, the Idylla showed no mutation detected in BRAF codon 600. Although none of these samples produced invalid or insufficient DNA input calls (even the sample with as low as $10 \%$ tumor cell content), it would be optimal to confirm their negative mutation status (wild-type) with another sensitive molecular method (e.g., pyrosequencing or droplet digital (dd) PCR) for further validation of the Idylla system. Simultaneously, it should be emphasized that the Idylla BRAF mutation assay has previously been shown to be able to accurately detect $B R A F$ mutational status in FFPE samples with even $2 \%$ tumor cell content [63].

In summary, we found a moderate BRAF V600E mutational frequency in our series of GGs and PAs, using a combination of BRAF-VE1 IHC on the Ventana stainer and realtime PCR-based BRAF mutation assay on the Idylla platform. We found this to be particularly accurate for pathology laboratories without molecular diagnostic units and in certain clinical situations demanding a rapid BRAF mutation analysis, given not only the similar merits of the assays but also the ability to complement each other's limitations. Both methods are CE-IVD labeled, easy to perform, fully automated, fast, cost-effective, and suitable for samples with a small quantity and low cellularity of tumor, as they have a low detection limit. BRAF-VE1 IHC appears even more suitable to characterize low-abundant tumor cells diluted in a large volume of the sample, but it detects only the V600E mutational variant, and the interpretation of staining results may be sometimes complicated and always requires the experienced pathologist. The Idylla BRAF mutation assay covers all the most prevalent and clinically relevant BRAF V600 mutations, and the interpretation of results is fully automatic. The present study showed a relatively good performance for BRAF-VE1 IHC in GG and PA with an occasional ambiguous or nonspecific staining in few samples, for which the Idylla molecular testing system allowed to determine the final results. Therefore, they seem to be complementary techniques also in the context of tissue-specific factors unique 
to brain tissue. However, their utility for neuropathology is highly dependent on validation of the Idylla technology on a larger cohort of CNS tumor patients against a reference method.

\section{Data Availability}

The datasets used and/or analyzed during the current study are available from the corresponding author on reasonable request.

\section{Conflicts of Interest}

The authors declare that they have no competing interests and conflicts of interest.

\section{Acknowledgments}

The study was supported by research tasks no. 141 within the framework of basal research activity (Nicolaus Copernicus University, Collegium Medicum, Bydgoszcz).

\section{References}

[1] Q. T. Ostrom, P. M. de Blank, C. Kruchko et al., "Alex’s Lemonade Stand Foundation Infant and Childhood Primary Brain and Central Nervous System Tumors Diagnosed in the United States in 2007-2011," Neuro-Oncology, vol. 16, supplement 10, pp. x1-x36, 2015.

[2] A. Elshahoubi, E. Khattab, H. Halalsheh, K. Khaleifeh, E. Bouffet, and N. Amayiri, "Feasibility of high-dose chemotherapy protocols to treat infants with malignant central nervous system tumors: experience from a middle-income country," Pediatric Blood \& Cancer, vol. 66, no. 1, article e27464, 2019.

[3] I. O. Gilli, A. F. Joaquim, H. Tedeschi, S. dos Santos Aguiar, A. M. Morcillo, and E. Ghizoni, "Factors affecting diagnosis of primary pediatric central nervous system neoplasias in a developing country," Child's Nervous System, vol. 35, no. 1, pp. 91-96, 2019.

[4] L. Bertero and P. Cassoni, Classification of tumours of the central nervous system, Springer, Cham, 2019.

[5] C. D. Kubicky, A. Sahgal, E. L. Chang, and S. S. Lo, "Rare primary central nervous system tumors," Rare Tumors, vol. 6, no. 3, pp. 105-110, 2017.

[6] M. Pekmezci, J. E. Villanueva-Meyer, B. Goode et al., “The genetic landscape of ganglioglioma," Acta Neuropathologica Communications, vol. 6, no. 1, p. 47, 2018.

[7] H. Ohgaki and P. Kleihues, "Population-based studies on incidence, survival rates, and genetic alterations in astrocytic and oligodendroglial gliomas," Journal of Neuropathology and Experimental Neurology, vol. 64, no. 6, pp. 479-489, 2005.

[8] V. P. Collins, D. T. W. Jones, and C. Giannini, "Pilocytic astrocytoma: pathology, molecular mechanisms and markers," Acta Neuropathologica, vol. 129, no. 6, pp. 775-788, 2015.

[9] H. Lavoie and M. Therrien, "Regulation of RAF protein kinases in ERK signalling," Nature Reviews Molecular Cell Biology, vol. 16, no. 5, pp. 281-298, 2015.

[10] L. R. Strickland, H. C. Pal, C. A. Elmets, and F. Afaq, "Targeting drivers of melanoma with synthetic small molecules and phytochemicals," Cancer Letters, vol. 359, no. 1, pp. 20-35, 2015.

[11] H. Davies, G. R. Bignell, C. Cox et al., "Mutations of the BRAF gene in human cancer," Nature, vol. 417, no. 6892, pp. 949954, 2002.

[12] L. Heinzerling, S. Kühnapfel, D. Meckbach et al., "Rare BRAF mutations in melanoma patients: implications for molecular testing in clinical practice," British Journal of Cancer, vol. 108, no. 10, pp. 2164-2171, 2013.

[13] P. Jain, A. Silva, H. J. Han et al., "Overcoming resistance to single-agent therapy for oncogenicBRAFgene fusionsviacombinatorial targeting of MAPK and PI3K/mTOR signaling pathways," Oncotarget, vol. 8, no. 49, pp. 84697-84713, 2017.

[14] C. Faulkner, H. P. Ellis, A. Shaw et al., "BRAF fusion analysis in pilocytic astrocytomas: KIAA1549-BRAF 15-9 fusions are more frequent in the midline than within the cerebellum," Journal of Neuropathology and Experimental Neurology, vol. 74, no. 9, pp. 867-872, 2015.

[15] T. T. Tomić, J. Olausson, A. Wilzén et al., "A new GTF2IBRAF fusion mediating MAPK pathway activation in pilocytic astrocytoma," PLoS One, vol. 12, no. 4, article e0175638, 2017.

[16] M. Gierke, J. Sperveslage, D. Schwab et al., "Analysis of IDH1R132 mutation, BRAF V600 mutation and KIAA1549-BRAF fusion transcript status in central nervous system tumors supports pediatric tumor classification," Journal of Cancer Research and Clinical Oncology, vol. 142, no. 1, pp. 89-100, 2016.

[17] M. Gessi and T. Pietsch, "The diagnostic role and clinical relevance of determination of BRAF status in brain tumors," Per Med, vol. 10, no. 4, pp. 405-412, 2013.

[18] B. H. Durham, B. Getta, S. Dietrich et al., "Genomic analysis of hairy cell leukemia identifies novel recurrent genetic alterations," Blood, vol. 130, no. 14, pp. 1644-1648, 2017.

[19] L. Cheng, A. Lopez-Beltran, F. Massari, G. T. MacLennan, and R. Montironi, "Molecular testing for _BRAF_ mutations to inform melanoma treatment decisions: a move toward precision medicine," Modern Pathology, vol. 31, no. 1, pp. 24-38, 2018.

[20] A. Czarniecka, M. Oczko-Wojciechowska, and M. Barczyński, "BRAF V600E mutation in prognostication of papillary thyroid cancer ( PTC ) recurrence," Gland Surgery, vol. 5, no. 5, pp. 495-505, 2016.

[21] A. C. Roden, X. Hu, S. Kip et al., "BRAF V600E expression in Langerhans cell histiocytosis," The American Journal of Surgical Pathology, vol. 38, no. 4, pp. 548-551, 2014.

[22] L. Zhu, C. Dong, Y. Cao et al., "Prognostic role of BRAF mutation in stage II/III colorectal cancer receiving curative resection and adjuvant chemotherapy : a meta-analysis based on randomized clinical trials," PLoS One, vol. 11, no. 5, article e0154795, 2016.

[23] A. Marranci, Z. Jiang, M. Vitiello et al., "The landscape of BRAF transcript and protein variants in human cancer," Molecular Cancer, vol. 16, no. 1, p. 85, 2017.

[24] E. R. Cantwell-Dorris, J. J. O'Leary, and O. M. Sheils, "BRAFV600E: implications for carcinogenesis and molecular therapy," Molecular Cancer Therapeutics, vol. 10, no. 3, pp. 385-394, 2011.

[25] D. Capper, A. S. Berghoff, M. Magerle et al., "Immunohistochemical testing of BRAF V600E status in 1,120 tumor tissue samples of patients with brain metastases," Acta Neuropathologica, vol. 123, no. 2, pp. 223-233, 2012. 
[26] F. Behling, A. Barrantes-Freer, M. Skardelly et al., "Frequency of BRAF V600E mutations in 969 central nervous system neoplasms," Diagnostic Pathology, vol. 11, no. 1, p. 55, 2016.

[27] D. Lee, Y. H. Cho, S. Y. Kang, N. Yoon, C. O. Sung, and Y. L. Suh, "BRAF V600E mutations are frequent in dysembryoplastic neuroepithelial tumors and subependymal giant cell astrocytomas," Journal of Surgical Oncology, vol. 111, no. 3, pp. 359-364, 2015.

[28] C. L. Penman, C. Faulkner, S. P. Lowis, and K. M. Kurian, "Current understanding of BRAF alterations in diagnosis, prognosis, and therapeutic targeting in pediatric low-grade gliomas," Frontiers in Oncology, vol. 5, 2015.

[29] P. Sadlecki, P. Antosik, D. Grzanka, M. Grabiec, and M. Walentowicz-Sadlecka, "KRAS mutation testing in borderline ovarian tumors and low-grade ovarian carcinomas with a rapid, fully integrated molecular diagnostic system," Tumor Biology, vol. 39, no. 10, article 101042831773398, 2017.

[30] P. Sadlecki, D. Grzanka, and M. Grabiec, “Testing forNRASmutations in serous borderline ovarian tumors and lowgrade serous ovarian carcinomas," Disease Markers, vol. 2018, 7 pages, 2018.

[31] M. C. Burger, M. W. Ronellenfitsch, N. I. Lorenz et al., "Dabrafenib in patients with recurrent, BRAF V600E mutated malignant glioma and leptomeningeal disease," Oncology Reports, vol. 38, no. 6, pp. 3291-3296, 2017.

[32] S. Dahiya, R. J. Emnett, D. H. Haydon et al., "BRAF-V600E mutation in pediatric and adult glioblastoma," Neuro-Oncology, vol. 16, no. 2, pp. 318-319, 2014.

[33] T. Kaley, M. Touat, V. Subbiah et al., "BRAF inhibition inBRAFV600-mutant gliomas: results from the VE-BASKET study," Journal of Clinical Oncology, vol. 36, no. 35, pp. 3477-3484, 2018.

[34] F. Behling and J. Schittenhelm, "Oncogenic BRAF alterations and their role in brain tumors," Cancers (Basel), vol. 11, no. 6, p. 794, 2019.

[35] G. Schindler, D. Capper, J. Meyer et al., "Analysis of BRAF V600E mutation in 1,320 nervous system tumors reveals high mutation frequencies in pleomorphic xanthoastrocytoma, ganglioglioma and extra-cerebellar pilocytic astrocytoma," Acta Neuropathologica, vol. 121, no. 3, pp. 397405, 2011.

[36] C. Chappé, L. Padovani, D. Scavarda et al., "Dysembryoplastic neuroepithelial tumors share with pleomorphic xanthoastrocytomas and gangliogliomas BRAFV600Emutation and expression," Brain Pathology, vol. 23, no. 5, pp. 574-583, 2013.

[37] M. J. Dougherty, M. Santi, M. S. Brose et al., "Activating mutations in BRAF characterize a spectrum of pediatric low-grade gliomas," Neuro-Oncology, vol. 12, no. 7, pp. 621-630, 2010.

[38] C. Koelsche, A. Wöhrer, A. Jeibmann et al., "Mutant BRAF $\mathrm{V} 600 \mathrm{E}$ protein in ganglioglioma is predominantly expressed by neuronal tumor cells," Acta Neuropathologica, vol. 125, no. 6, pp. 891-900, 2013.

[39] J. K. Myung, H. Cho, C.-K. Park, S.-K. Kim, S.-H. Lee, and S.H. Park, "Analysis of the BRAF ${ }^{\mathrm{V} 600 \mathrm{E}}$ Mutation in Central Nervous System Tumors," Translational Oncology, vol. 5, no. 6, pp. 430-436, 2012.

[40] Q. Breton, H. Plouhinec, D. Prunier-Mirebeau et al., "BRAF-V600E immunohistochemistry in a large series of glial and glial-neuronal tumors," Brain and Behavior: A Cognitive Neuroscience Perspective, vol. 7, no. 3, p. e00641, 2017.
[41] L. Schirosi, S. Strippoli, F. Gaudio et al., "Is immunohistochemistry of BRAF V600E useful as a screening tool and during progression disease of melanoma patients?," BMC Cancer, vol. 16, no. 1, pp. 905-911, 2016.

[42] M. Szymonek, A. Kowalik, J. Kopczyński et al., "Immunohistochemistry cannot replace DNA analysis for evaluation ofBRAFV600E mutations in papillary thyroid carcinoma," Oncotarget, vol. 8, no. 43, pp. 74897-74909, 2017.

[43] S. Brandner and A. von Deimling, "Invited review: diagnostic, prognostic and predictive relevance of molecular markers in gliomas," Neuropathology and Applied Neurobiology, vol. 41, no. 6, pp. 694-720, 2015.

[44] J. Kim, D. Kim, J. S. Lim et al., "The use of technical replication for detection of low-level somatic mutations in nextgeneration sequencing," Nature Communications, vol. 10, no. 1, 2019.

[45] P.-J. Lamy, F. Castan, N. Lozano et al., "Next-Generation Genotyping by Digital PCR to Detect and Quantify the _BRAF_ V600E Mutation in Melanoma Biopsies," The Journal of Molecular Diagnostics, vol. 17, no. 4, pp. 366-373, 2015.

[46] M. Elazezy and S. A. Joosse, "Techniques of using circulating tumor DNA as a liquid biopsy component in cancer management," Computational and Structural Biotechnology Journal, vol. 16, pp. 370-378, 2018.

[47] D. Capper, M. Preusser, A. Habel et al., “Assessment of BRAF V600E mutation status by immunohistochemistry with a mutation-specific monoclonal antibody," Acta Neuropathologica, vol. 122, no. 1, pp. 11-19, 2011.

[48] K. Dvorak, A. Higgins, J. Palting, M. Cohen, and P. Brunhoeber, "Immunohistochemistry with anti-BRAF V600E (VE1) mouse monoclonal antibody is a sensitive method for detection of the BRAF V600E mutation in colon cancer: evaluation of 120 cases with and without KRAS mutation and literature review," Pathology Oncology Research, vol. 25, no. 1, pp. 349-359, 2019.

[49] M. V. Pearlstein, D. C. Zedek, D. W. Ollila et al., "Validation of the VE1 immunostain for the BRAF V600E mutation in melanoma," Journal of Cutaneous Pathology, vol. 41, no. 9, pp. 724732, 2014.

[50] G. V. Long, J. S. Wilmott, D. Capper et al., "Immunohistochemistry is highly sensitive and specific for the detection of V600E BRAF mutation in melanoma," The American Journal of Surgical Pathology, vol. 37, no. 1, pp. 61-65, 2013.

[51] M. I. Ilie, S. Lassalle, E. Long-Mira et al., "Diagnostic value of immunohistochemistry for the detection of the BRAF V600E mutation in papillary thyroid carcinoma: comparative analysis with three DNA-based assays," Thyroid, vol. 24, no. 5, pp. 858866, 2014.

[52] P. A. McKelvie, F. Chan, Y. Yu et al., "The prognostic significance of the BRAF ${ }^{\mathrm{V} 600 \mathrm{E}}$ mutation in papillary thyroid carcinoma detected by mutation-specific immunohistochemistry," Pathology, vol. 45, no. 7, pp. 637-644, 2013.

[53] J. S. Estrella, M. T. Tetzlaff, R. L. Bassett et al., "Assessment of BRAF V600E status in colorectal carcinoma: tissue-specific discordances between immunohistochemistry and sequencing," Molecular Cancer Therapeutics, vol. 14, no. 12, pp. 2887-2895, 2015.

[54] B. K. Kleinschmidt-DeMasters, D. L. Aisner, and N. K. Foreman, "BRAF VE1 immunoreactivity patterns in epithelioid glioblastomas positive for BRAF V600E mutation," The American Journal of Surgical Pathology, vol. 39, no. 4, pp. 528-540, 2015. 
[55] Z. Tosuner, M. Gecer, M. Hatiboğlu, A. Abdallah, and S. Turna, "BRAF V600E mutation and BRAF VE1 immunoexpression profiles in different types of glioblastoma," Oncology Letters, vol. 16, no. 2, pp. 2402-2408, 2018.

[56] S. F. Kuan, S. Navina, K. L. Cressman, and R. K. Pai, "Immunohistochemical detection of BRAF V600E mutant protein using the VE1 antibody in colorectal carcinoma is highly concordant with molecular testing but requires rigorous antibody optimization," Human Pathology, vol. 45, no. 3, pp. 464-472, 2014.

[57] E. Q. Lee, S. Ruland, N. R. LeBoeuf, P. Y. Wen, and S. Santagata, "Successful treatment of a ProgressiveBRAFV600E-Mutated anaplastic pleomorphic xanthoastrocytoma with vemurafenib monotherapy," Journal of Clinical Oncology, vol. 34, no. 10, pp. e87-e89, 2016.

[58] I. M. Løes, H. Immervoll, J. H. Angelsen et al., "Performance comparison of three BRAF V600E detection methods in malignant melanoma and colorectal cancer specimens," Tumor Biology, vol. 36, no. 2, pp. 1003-1013, 2015.

[59] Simple IS Idylla ${ }^{\mathrm{TM}}$, "A Revolutionary, fully automated system that makes molecular testing convenient and exceptionally fast. Suitable for any lab," https://www.biocartis.com/en/ meet-idylla/idylla-platform.

[60] T. Idylla, "Mutation B, System I. Technical sheet idylla ${ }^{\mathrm{TM}}$ BRAF mutation test. 5-7," https://www.biocartis.com/en/meetidylla/idylla-platform.

[61] S. Maraka and F. Janku, "BRAF alterations in primary brain tumors," Discovery Medicine, vol. 26, no. 141, pp. 51-60, 2018.

[62] J. Solassol, J. Vendrell, B. Märkl et al., "Multi-center evaluation of the fully automated PCR-based Idylla ${ }^{\mathrm{TM}}$ KRAS mutation assay for rapid KRAS mutation status determination on formalin-fixed paraffin-embedded tissue of human colorectal cancer," PLoS One, vol. 11, no. 9, article e0163444, 2016.

[63] C. Bisschop, A. ter Elst, L. J. Bosman et al., "Rapid BRAF mutation tests in patients with advanced melanoma: comparison of immunohistochemistry, droplet digital PCR, and the Idylla mutation platform," Melanoma Research, vol. 28, no. 2, pp. 96-104, 2018.

[64] A. Harlé, J. Salleron, C. Franczak et al., "Detection of BRAF mutations using a fully automated platform and comparison with high resolution melting, real-time allele specific amplification, immunohistochemistry and next generation sequencing assays, for patients with metastatic melanoma," PLoS One, vol. 11, no. 4, article e0153576, 2016.

[65] M. K. Yeo, M. K. Jung, S. Y. Lee, Y. M. Lee, G. M. Hur, and J. M. Kim, "The usefulness of a novel fully automated PCRbased Idylla test for detection of the BRAF V600E mutation in thyroid tissue: comparison with PNA-clamping PCR, realtime PCR and pyrosequencing," Journal of Clinical Pathology, vol. 70, no. 3, pp. 260-265, 2017.

[66] R. Colling, L. M. Wang, and E. Soilleux, "Automated PCR detection of BRAF mutations in colorectal adenocarcinoma: a diagnostic test accuracy study," Journal of Clinical Pathology, vol. 69, no. 5, pp. 398-402, 2016.

[67] A.-I. Schiefer, L. Parlow, L. Gabler et al., "Multicenter Evaluation of a Novel Automated Rapid Detection System of _BRAF_ Status in Formalin-Fixed, Paraffin-Embedded Tissues," The Journal of Molecular Diagnostics, vol. 18, no. 3, pp. 370-377, 2016. 